\title{
ON EXTRINSICALLY SYMMETRIC HYPERSURFACES OF $\mathbb{H}^{n} \times \mathbb{R}$
}

\author{
GIOVANNI CALVARUSO, DANIEL KOWALCZYK and JOERI VAN DER VEKEN ${ }^{凶}$
}

\author{
(Received 13 February 2009)
}

\begin{abstract}
Totally umbilical, semi-parallel and parallel hypersurfaces of $\mathbb{H}^{n} \times \mathbb{R}$ are completely classified. More examples arise than in the analogous study on the ambient space $\mathbb{S}^{n} \times \mathbb{R}$.
\end{abstract}

2000 Mathematics subject classification: primary 53B25; secondary 53C40.

Keywords and phrases: hypersurface, parallel, semi-parallel, totally umbilical.

\section{Introduction}

Given a Riemannian manifold, it is an interesting problem to study and classify its hypersurfaces endowed by special symmetry properties. Natural extrinsic symmetry properties are total geodesy, total umbilicity, parallelism and semi-parallelism. Such a study enriches our understanding of the geometry of the ambient space and permits a comparison with analogous results obtained in different ambient spaces.

Real space forms are clearly the first candidates for the study of their hypersurfaces, since their curvature tensor takes the simplest possible form. It is well known that totally geodesic and totally umbilical hypersurfaces of a real space form are extrinsic spheres end hence are all parallel. A complete classification of parallel and semiparallel hypersurfaces in real space forms can respectively be found in [5] and in [3].

Besides real space forms, the Riemannian manifolds with the simplest curvature tensor are the remaining conformally flat symmetric spaces, that is, the Riemannian products $\mathbb{S}^{n}(\kappa) \times \mathbb{R}, \mathbb{H}^{n}(-\kappa) \times \mathbb{R}$ and $\mathbb{S}^{p}(\kappa) \times \mathbb{H}^{q}(-\kappa)$; see [6]. It is now natural to consider these as ambient spaces and to try to classify their hypersurfaces endowed with special extrinsic symmetry properties. This study was started by the third named author and Vrancken in [9], where hypersurfaces of $\mathbb{S}^{n} \times \mathbb{R}$ were investigated. One of the interesting differences with respect to the case where the ambient space is a real space form is that total umbilicity does not imply parallelism. In the present paper,

The third named author is a postdoctoral researcher supported by the Research Foundation-Flanders (FWO).

(C) 2010 Australian Mathematical Publishing Association Inc. 0004-9727/2010 \$16.00 
totally geodesic, totally umbilical, parallel and semi-parallel hypersurfaces of $\mathbb{H}^{n} \times \mathbb{R}$ will be completely classified, and their relationship with rotation hypersurfaces will be explained. It is worthwhile to note that more examples arise in $\mathbb{H}^{n} \times \mathbb{R}$ than in the analogous study on the ambient space $\mathbb{S}^{n} \times \mathbb{R}$.

The paper is organized as follows. In Section 2 we will provide a basic description of a hypersurface of $\mathbb{H}^{n} \times \mathbb{R}$, also recalling the definition and properties of a rotation hypersurface in $\mathbb{H}^{n} \times \mathbb{R}$. Totally umbilical, semi-parallel and parallel hypersurfaces of $\mathbb{H}^{n} \times \mathbb{R}$ will be classified in Sections 3, 4 and 5, respectively.

\section{Preliminaries}

Let $F: M^{n} \rightarrow \widetilde{M}^{n+1}$ be an isometric immersion of (semi-)Riemannian manifolds with Levi-Civita connections $\nabla$ and $\widetilde{\nabla}$, respectively. We will always assume manifolds to be smooth and connected. Vector fields tangent to $M^{n}$ will be denoted by $X, Y, Z, \ldots$ and a unit normal vector field on $M^{n}$ in $\widetilde{M}^{n+1}$ will usually be denoted by $N$. The formulae of Gauss and Weingarten, which concern the decomposition of the vector fields $\widetilde{\nabla}_{X} Y$ and $\widetilde{\nabla}_{X} N$ into their tangential and normal components along the submanifold $M^{n}$, are given by (see [1])

$$
\begin{gathered}
\widetilde{\nabla}_{X} Y=\nabla_{X} Y+h(X, Y) N, \\
\widetilde{\nabla}_{X} N=-S X .
\end{gathered}
$$

Here, $h$ is a symmetric $(0,2)$-tensor field, called the scalar-valued second fundamental form of the hypersurface, and $S$ is a symmetric $(1,1)$-tensor field, called the shape operator associated with $N$, such that $h(X, Y)=\langle S X, Y\rangle$. Moreover, the equations of Gauss and Codazzi are given respectively by

$$
\begin{gathered}
\langle\widetilde{R}(X, Y) Z, W\rangle=\langle R(X, Y) Z, W\rangle+h(X, Z) h(Y, W)-h(X, W) h(Y, Z), \\
\langle\widetilde{R}(X, Y) Z, \xi\rangle=(\nabla h)(X, Y, Z)-(\nabla h)(Y, X, Z),
\end{gathered}
$$

where $R$ and $\widetilde{R}$ are the Riemann-Christoffel curvature tensors of $M^{n}$ and $\widetilde{M}^{n+1}$ and the covariant derivative of $h$ is defined by

$$
(\nabla h)(X, Y, Z)=X[h(Y, Z)]-h\left(\nabla_{X} Y, Z\right)-h\left(Y, \nabla_{X} Z\right) .
$$

A totally geodesic hypersurface $M^{n}$ is a hypersurface for which $h=0$. A hypersurface $M^{n}$ is totally umbilical if $h$ is a scalar multiple of the metric at every point, that is, $h(X, Y)=\lambda\langle X, Y\rangle$ for some function $\lambda$ on $M^{n}$. A hypersurface $M^{n}$ is called parallel if $\nabla h=0$ and semi-parallel if $R \cdot h=0$, where

$$
(R \cdot h)(X, Y, Z, W)=-h(R(X, Y) Z, W)-h(Z, R(X, Y) W) .
$$

Let

$$
\mathbb{L}^{n+2}=\left(\mathbb{R}^{n+2},-d x_{1}^{2}+d x_{2}^{2}+\cdots+d x_{n+2}^{2}\right)
$$


be the flat Lorentzian space of dimension $n+2$ and define $\mathbb{H}^{n} \times \mathbb{R}$ as the following subset of $\mathbb{L}^{n+2}$, equipped with the induced metric:

$$
\mathbb{H}^{n} \times \mathbb{R}=\left\{\left(x_{1}, \ldots, x_{n+2}\right) \in \mathbb{L}^{n+2} \mid-x_{1}^{2}+x_{2}^{2}+\cdots+x_{n+1}^{2}=-1, x_{1}>0\right\} .
$$

Then $\mathbb{H}^{n} \times \mathbb{R}$ is the Riemannian product of the hyperbolic space $\mathbb{H}^{n}$ of constant sectional curvature -1 and the real line, and $\xi=\left(x_{1}, \ldots, x_{n+1}, 0\right)$ is a normal vector field on $\mathbb{H}^{n} \times \mathbb{R}$ in $\mathbb{L}^{n+2}$ satisfying $\langle\xi, \xi\rangle=-1$. We denote by $X_{\mathbb{H}^{n}}$ the projection of a vector field $X$ tangent to $\mathbb{H}^{n} \times \mathbb{R}$ onto the tangent space of $\mathbb{H}^{n}$, that is, $X_{\mathbb{H}^{n}}=X-\left\langle X, \partial_{n+2}\right\rangle \partial_{n+2}$. Using Gauss's formula for isometric immersions in semi-Riemannian manifolds, we find that the Levi-Civita connection $\widetilde{\nabla}$ of $\mathbb{H}^{n} \times \mathbb{R}$ is given by

$$
\widetilde{\nabla}_{X} Y=D_{X} Y-\left\langle X_{\mathbb{H}^{n}}, Y_{\mathbb{H}^{n}}\right\rangle \xi,
$$

where $X, Y$ are vector fields tangent to $\mathbb{H}^{n} \times \mathbb{R}$ and $D$ is the Levi-Civita connection of $\mathbb{L}^{n+2}$. Consequently, we obtain that the Riemann-Christoffel curvature tensor $\widetilde{R}$ of $\mathbb{H}^{n} \times \mathbb{R}$ is given by

$$
\langle\widetilde{R}(X, Y) Z, W\rangle=\left\langle X_{\mathbb{H}^{n}}, Z_{\mathbb{H}^{n}}\right\rangle\left\langle Y_{\mathbb{H}^{n}}, W_{\mathbb{H}^{n}}\right\rangle-\left\langle Y_{\mathbb{H}^{n}}, Z_{\mathbb{H}^{n}}\right\rangle\left\langle X_{\mathbb{H}^{n}}, W_{\mathbb{H}^{n}}\right\rangle .
$$

Consider a hypersurface $F: M^{n} \rightarrow \mathbb{H}^{n} \times \mathbb{R}$ with unit normal $N$. Let $\nabla$ and $R$ be the Levi-Civita connection and the Riemann-Christoffel curvature tensor of $M^{n}$. Denote by $T$ the projection of the vector field $\partial_{n+2}$ onto the tangent space to $M^{n}$ and denote by $\theta$ a function on $M^{n}$ such that $\cos \theta=\left\langle N, \partial_{n+2}\right\rangle$. So we obtain that $\partial_{n+2}=T+\cos \theta N$ along $M^{n}$. Using these notations and Equation (2.9), the equations of Gauss and Codazzi, (2.3) and (2.4), reduce to

$$
\begin{array}{rl}
\langle R(X, Y) Z, W\rangle=\langle S & X, W\rangle\langle S Y, Z\rangle-\langle S X, Z\rangle\langle S Y, W\rangle \\
& -\langle X, W\rangle\langle Y, Z\rangle+\langle X, Z\rangle\langle Y, W\rangle \\
& -\langle Y, T\rangle\langle W, T\rangle\langle X, Z\rangle-\langle X, T\rangle\langle Z, T\rangle\langle Y, W\rangle \\
& +\langle X, T\rangle\langle W, T\rangle\langle Y, Z\rangle+\langle Y, T\rangle\langle Z, T\rangle\langle X, W\rangle, \\
\nabla_{X} S Y-\nabla_{Y} & S X-S[X, Y]=\cos \theta(\langle X, T\rangle Y-\langle Y, T\rangle X),
\end{array}
$$

where $X, Y, Z$ and $W$ are vector fields tangent to $M^{n}$. Using the fact that $\partial_{n+2}$ is parallel in $\mathbb{H}^{n} \times \mathbb{R}$, we also obtain the following equations:

$$
\begin{gathered}
\nabla_{X} T=\cos \theta S X, \\
X[\cos \theta]=-\langle S X, T\rangle .
\end{gathered}
$$

These equations appear in the following existence and uniqueness theorem for isometric immersions of hypersurfaces of $\mathbb{H}^{n} \times \mathbb{R}$.

THEOREM 2.1 [2]. Let $M^{n}$ be a simply connected Riemannian manifold with LeviCivita connection $\nabla$ and curvature tensor $R$. Let $S$ be a field of symmetric operators $S_{p}: T_{p} M^{n} \rightarrow T_{p} M^{n}$, and let $T$ and $\theta$ be a vector field and a smooth function on $M^{n}$ 
such that $\|T\|^{2}=\sin ^{2} \theta$. Assume that Equations (2.10)-(2.13) are satisfied. Then there exists an isometric immersion $F: M^{n} \rightarrow \mathbb{H}^{n} \times \mathbb{R}$ with unit normal $N$, such that the shape operator with respect to this normal is given by $S$ and such that $\partial_{n+2}=T+\cos \theta N$. Moreover, the immersion is unique up to global isometries of $\mathbb{H}^{n} \times \mathbb{R}$ preserving the orientations of both $\mathbb{H}^{n}$ and $\mathbb{R}$.

We will now recall the definition of a rotation hypersurface of $\mathbb{H}^{n} \times \mathbb{R}$, as proposed in [4]. Consider a three-dimensional subspace $P^{3}$ of $\mathbb{L}^{n+2}$ containing the $x_{n+2}$-axis. Then $\left(\mathbb{H}^{n} \times \mathbb{R}\right) \cap P^{3}=\mathbb{H}^{1} \times \mathbb{R}$. Let $P^{2}$ be a two-dimensional subspace of $P^{3}$, also containing the $x_{n+2}$-axis. Let $\mathcal{I}$ denote the group of isometries of $\mathbb{L}^{n+2}$, which leave $\mathbb{H}^{n} \times \mathbb{R}$ globally invariant and which leave the subspace $P^{2}$ pointwise fixed. Let $\alpha$ be a curve in $\mathbb{H}^{1} \times \mathbb{R}$ which does not intersect $P^{2}$. Then the rotation hypersurface $M^{n}$ of $\mathbb{H}^{n} \times \mathbb{R}$ with profile curve $\alpha$ and axis $P^{2}$ is defined as the $\mathcal{I}$-orbit of $\alpha$. From the definition it follows that the velocity vector of $\alpha$ is proportional to $T$, unless $\alpha$ lies in a plane orthogonal to $\partial_{n+2}$, in which case $T=0$. We will consider three cases, depending on whether $P^{2}$ is Lorentzian, Riemannian or degenerate. Without loss of generality, we will assume in the following that $P^{3}$ is spanned by $\left\{\partial_{1}, \partial_{n+1}, \partial_{n+2}\right\}$ and that $P^{2}$ is spanned by $\left\{\partial_{1}, \partial_{n+2}\right\}$ (Lorentzian), by $\left\{\partial_{n+1}, \partial_{n+2}\right\}$ (Riemannian) or by $\left\{e_{n+1}, e_{n+2}\right\}$ (degenerate), where $\left\{e_{1}, \ldots, e_{n+2}\right\}$ is a pseudo-orthonormal basis of $\mathbb{L}^{n+2}$ with

$$
e_{1}=\frac{1}{\sqrt{2}}\left(\partial_{1}+\partial_{n+1}\right), \quad e_{n+1}=\frac{1}{\sqrt{2}}\left(-\partial_{1}+\partial_{n+1}\right), \quad e_{k}=\partial_{k}
$$

for $k \in\{2, \ldots, n, n+2\}$. It was proven in [4] that there exists a local orthonormal frame $\left\{f_{1}, \ldots, f_{n}\right\}$ on a rotation hypersurface $M^{n}$, with $T=\|T\| f_{1}$, such that the shape operator $S$ takes the following diagonal form with respect to $\left\{f_{1}, \ldots, f_{n}\right\}$ :

$$
S=\left(\begin{array}{cccc}
\lambda & & & \\
& \mu & & \\
& & \ddots & \\
& & & \mu
\end{array}\right) .
$$

The principal curvatures $\lambda$ and $\mu$ are constant on orbits and can be computed explicitly as follows. Assume first that $P^{2}$ is either Riemannian or Lorentzian. If the profile curve $\alpha$ is not a vertical line in $\mathbb{H}^{n} \times \mathbb{R}$, it can be locally parametrized as $\alpha(s)=$ $(\cosh s, 0, \ldots, 0, \sinh s, a(s))$ and we have that

$$
\lambda=-\frac{a^{\prime \prime}(s)}{\left(1+a^{\prime}(s)^{2}\right)^{3 / 2}}, \quad \mu=-\frac{a^{\prime}(s) \operatorname{coth} s}{\left(1+a^{\prime}(s)^{2}\right)^{1 / 2}}
$$

if $P^{2}=\operatorname{span}\left\{\partial_{1}, \partial_{n+2}\right\}$ is Lorentzian, and

$$
\lambda=-\frac{a^{\prime \prime}(s)}{\left(1+a^{\prime}(s)^{2}\right)^{3 / 2}}, \quad \mu=-\frac{a^{\prime}(s) \tanh s}{\left(1+a^{\prime}(s)^{2}\right)^{1 / 2}}
$$


if $P^{2}=\operatorname{span}\left\{\partial_{n+1}, \partial_{n+2}\right\}$ is Riemannian. If $\alpha$ is a vertical line $\alpha(s)=(\cosh c$, $0, \ldots, 0, \sinh c, s)$, with $c \in \mathbb{R}$, we have that

$$
\begin{array}{ll}
\lambda=0, \quad \mu=-\operatorname{coth} c & \text { if } P^{2}=\operatorname{span}\left\{\partial_{1}, \partial_{n+2}\right\} \text { is Lorentzian, } \\
\lambda=0, \quad \mu=-\tanh c \quad \text { if } P^{2}=\operatorname{span}\left\{\partial_{n+1}, \partial_{n+2}\right\} \text { is Riemannian. }
\end{array}
$$

Next, assume that the axis of the rotation hypersurface is degenerate. If the profile curve is not a vertical line in $\mathbb{H}^{n} \times \mathbb{R}$, it can be locally parametrized as $\alpha(s)=(s, 0, \ldots, 0,-1 / 2 s, a(s))$ with respect to the pseudo-orthonormal basis $\left\{e_{1}, \ldots, e_{n+2}\right\}$ defined by (2.14) and we obtain that

$$
\lambda=-\frac{s a^{\prime}(s)+s^{2} a^{\prime \prime}(s)}{\left(1+s^{2} a^{\prime}(s)\right)^{3 / 2}}, \quad \mu=-\frac{s a^{\prime}(s)}{\left(1+s^{2} a^{\prime}(s)\right)^{1 / 2}} .
$$

If $\alpha$ is a vertical line $\alpha(s)=(c, 0, \ldots, 0,-1 / 2 c, s)$, with $c \in \mathbb{R}$, we obtain

$$
\lambda=0, \quad \mu=-1 \text {. }
$$

Finally, we also mention the following characterization of rotation hypersurfaces of $\mathbb{H}^{n} \times \mathbb{R}$.

THEOREM 2.2 [4]. Let $n \geq 3$ and let $F: M^{n} \rightarrow \mathbb{H}^{n} \times \mathbb{R}$ be a hypersurface with shape operator

$$
S=\left(\begin{array}{llll}
\lambda & & & \\
& \mu & & \\
& & \ddots & \\
& & & \mu
\end{array}\right),
$$

with $\lambda \neq \mu$ and suppose that $S T=\lambda T$. Assume, moreover, that there is a functional relation $\lambda(\mu)$. Then $M^{n}$ is an open part of a rotation hypersurface.

\section{Totally umbilical hypersurfaces}

In this section, we give a complete classification of totally umbilical hypersurfaces in $\mathbb{H}^{n} \times \mathbb{R}$ and we will see that, unlike for real space forms, the ratio between the second fundamental form and the metric can be nonconstant. We remark that the third author has classified totally umbilical surfaces in $\mathbb{S}^{2} \times \mathbb{R}$ and $\mathbb{H}^{2} \times \mathbb{R}$, by means of an explicit parametrization, in [8]. Independently, another description of the same family of surfaces was obtained in [7]. Moreover, together with Vrancken, the third author classified totally umbilical hypersurfaces in $\mathbb{S}^{n} \times \mathbb{R}$ in [9]. We will see that in the case of $\mathbb{H}^{n} \times \mathbb{R}$, more families of totally umbilical hypersurfaces arise.

First, we classify totally geodesic hypersurfaces. The proof of the following theorem is based on Codazzi's equation and is analogous to the proof of the corresponding theorem in [9].

THEOREM 3.1. Let $M^{n}$ be a totally geodesic hypersurface of $\mathbb{H}^{n} \times \mathbb{R}$. Then $M^{n}$ is an open part of a hypersurface $\mathbb{H}^{n} \times\left\{t_{0}\right\}$ for $t_{0} \in \mathbb{R}$, or of a hypersurface $\bar{M}^{n-1} \times \mathbb{R}$, with $\bar{M}^{n-1}$ a totally geodesic hypersurface of $\mathbb{H}^{n}$. 
The following proposition can also be proven in a similar way to its counterpart in [9].

PROPOSITION 3.2. Let $M^{n}$ be a totally umbilical hypersurface of $\mathbb{H}^{n} \times \mathbb{R}$ with angle function $\theta$, structure vector field $T$ and shape operator $S$ and let $p$ be a point of $M^{n}$ such that $\sin \theta(p) \neq 0$. Then there exist local coordinates $\left(u, v_{1}, \ldots, v_{n-1}\right)$ on $a$ neighbourhood of $p$ in $M^{n}$, such that $\theta$ only depends on $u, T=\sin \theta \partial_{u}, S=\theta^{\prime} \mathrm{Id}$ and the following equation holds:

$$
\phi^{\prime \prime}-\sin \phi=0 \quad \text { with } \phi=2 \theta .
$$

Conversely, starting with an open subset $U \subseteq \mathbb{R}^{n}$ with coordinates $\left(u, v_{1}, \ldots, v_{n-1}\right)$ and a solution $\phi(u)$ of (3.1), such that $\sin (\phi / 2)$ vanishes nowhere on $U$, we can define a Riemannian metric on $U$ such that there exists a totally umbilical isometric immersion $F: U \rightarrow \mathbb{H}^{n} \times \mathbb{R}$ with angle function $\theta=\phi / 2$.

We can now prove the classification of totally umbilical hypersurfaces of $\mathbb{H}^{n} \times \mathbb{R}$.

THEOREM 3.3. Let $M^{n}$ be a totally umbilical hypersurface of $\mathbb{H}^{n} \times \mathbb{R}$, which is not totally geodesic, with angle function $\theta$ and let $p$ be a point of $M^{n}$ such that $\sin \theta(p) \neq 0$. Then there exists a local coordinate system $\left(u, v_{1}, \ldots, v_{n-1}\right)$ on $M^{n}$ such that $\theta$ only depends on $u$, the shape operator is $S=\theta^{\prime} \mathrm{Id}$ and

$$
\left(\theta^{\prime}\right)^{2}-\sin ^{2} \theta=c
$$

where $c>-1$ is a real constant. Moreover, $M^{n}$ is locally congruent to a rotation hypersurface of $\mathbb{H}^{n} \times \mathbb{R}$ for which the profile curve $\alpha$ and the axis $P^{2}$ are given by:

- $\quad \alpha(u)=(1 / \sqrt{c})\left(\theta^{\prime}, 0, \ldots, 0, \sin \theta, \sqrt{c} \int \sin \theta d u\right), P^{2}=\operatorname{span}\left\{\partial_{1}, \partial_{n+2}\right\}$, if $c>0$;

- $\alpha(u)=(1 / \sqrt{-c})\left(\sin \theta, 0, \ldots, 0, \theta^{\prime}, \sqrt{-c} \int \sin \theta d u\right), P^{2}=\operatorname{span}\left\{\partial_{n+1}, \partial_{n+2}\right\}$, if $0>c>-1$;

- $\quad \alpha(u)=\left(\theta^{\prime}, 0, \ldots, 0,-1 / 2 \theta^{\prime}, \int \sin \theta d u\right)$ with respect to the pseudo-orthonormal basis $\left\{e_{1}, \ldots, e_{n+2}\right\}, P^{2}=\operatorname{span}\left\{e_{n+1}, e_{n+2}\right\}$, if $c=0$.

Conversely, all rotation hypersurfaces with profile curves and axes given above, where $\theta$ and c satisfy (3.2), are totally umbilical in $\mathbb{H}^{n} \times \mathbb{R}$.

PROOF. Let $M^{n}$ be a totally umbilical hypersurface of $\mathbb{H}^{n} \times \mathbb{R}$, which is not totally geodesic, with shape operator $S=\lambda \mathrm{Id}$ and angle function $\theta$. It follows from Proposition 3.2 that there exists a local coordinate system $\left(u, v_{1}, \ldots, v_{n-1}\right)$ on $U \subseteq M^{n}$ such that $\theta$ only depends on $u$ and $\left(\theta^{\prime}\right)^{2}-\sin ^{2} \theta=c$ is a constant, this last equation being a direct consequence of Equation (3.1). Remark that $c \geq-1$ and that $c=-1$ if and only if $\cos \theta=0$ everywhere, that is, if and only if $M^{n}$ is an open part of $\bar{M}^{n-1} \times \mathbb{R}$ with $\bar{M}^{n-1}$ a hypersurface of $\mathbb{H}^{n}$. It is easily verified that $\bar{M}^{n-1} \times \mathbb{R}$ is a totally umbilical hypersurface of $\mathbb{H}^{n} \times \mathbb{R}$ if and only if it is a totally geodesic hypersurface. This case was excluded in the formulation of the theorem and we conclude that $c>1$. 
By Proposition 3.2, we have $T=\sin \theta \partial_{u}$ and $\lambda=\theta^{\prime}$. A direct computation, using the structure Equations (2.10)-(2.13), shows that the induced metric on $U$ is $g=d u^{2}+\sin ^{2} \theta g_{c}\left(v_{1}, \ldots, v_{n-1}\right)$, where $g_{c}$ is a Riemannian metric of constant sectional curvature $c$. Due to the uniqueness part of Theorem 2.1, it is now sufficient to give an immersion $F: U \rightarrow \mathbb{H}^{n} \times \mathbb{R}$, such that $F$ is isometric, the projection of $\partial_{n+2}$ on $F(U)$ is $F_{*}\left(\sin \theta \partial_{u}\right)$, the angle between the unit normal $N$ and $\partial_{n+2}$ is $\theta$ and the shape operator $S=\theta^{\prime}$ Id. We consider three cases depending on whether $c>0,0>c>-1$ or $c=0$.

If $c>0$, we consider the immersion

$$
F\left(u, v_{1}, \ldots, v_{n-1}\right)=\frac{1}{\sqrt{c}}\left(\theta^{\prime}, \phi_{1} \sin \theta, \ldots, \phi_{n} \sin \theta, \sqrt{c} \int \sin \theta d u\right),
$$

where $\left(\phi_{1}\left(v_{1}, \ldots, v_{n-1}\right), \ldots, \phi_{n}\left(v_{1}, \ldots, v_{n-1}\right)\right)$ is a parametrization of $\mathbb{S}^{n-1}(1)$ in $\mathbb{E}^{n}$. It is easily verified by a straightforward calculation that $F$ satisfies the necessary conditions. Remark that $F$ is a parametrization of a rotation hypersurface of $\mathbb{H}^{n} \times \mathbb{R}$ with axis $P^{2}=\operatorname{span}\left\{\partial_{1}, \partial_{n+2}\right\}$ and the profile curve given in the first case of the theorem.

If $0>c>-1$, we see that the immersion

$$
F\left(u, v_{1}, \ldots, v_{n-1}\right)=\frac{1}{\sqrt{-c}}\left(\phi_{1} \sin \theta, \ldots, \phi_{n} \sin \theta, \theta^{\prime}, \sqrt{-c} \int \sin \theta d u\right),
$$

where $\left(\phi_{1}\left(v_{1}, \ldots, v_{n-1}\right), \ldots, \phi_{n}\left(v_{1}, \ldots, v_{n-1}\right)\right)$ is a parametrization of $\mathbb{H}^{n-1}(-1)$ in $\mathbb{L}^{n}$, satisfies the necessary conditions. This corresponds to the second case of the theorem.

Finally, for $c=0$, we consider the immersion

$$
F\left(u, v_{1}, \ldots, v_{n-1}\right)=\left(\theta^{\prime}, \theta^{\prime} v_{1}, \ldots, \theta^{\prime} v_{n-1},-\frac{1}{2 \theta^{\prime}}-\frac{\theta^{\prime}}{2}\left(\sum_{i=1}^{n-1} v_{i}^{2}\right), \int \sin \theta d u\right),
$$

with respect to the pseudo-orthonormal basis $\left\{e_{1}, \ldots, e_{n+2}\right\}$. It follows again by a straightforward calculation that $F$ satisfies the necessary conditions and that this corresponds to the third case of the theorem.

\section{Semi-parallel hypersurfaces of $\mathbb{H}^{n} \times \mathbb{R}$}

In this section we will classify the semi-parallel hypersurfaces of $\mathbb{H}^{n} \times \mathbb{R}$. Again, if we compare our result to the classification in $\mathbb{S}^{n} \times \mathbb{R}$, given in [9], some new interesting families arise.

The following lemma characterizes the semi-parallel hypersurfaces in terms of their shape operators and can be proven in the same way as its spherical counterpart in [9].

LEMMA 4.1. Let $M^{n}$ be a semi-parallel hypersurface of $\mathbb{H}^{n} \times \mathbb{R}$. Let $T$ and $\theta$ be as above. Then there exists a local orthonormal frame field $\left\{f_{1}, \ldots, f_{n}\right\}$ on $M^{n}$ with respect to which the shape operator takes one of the following forms: 
(1) $S=\lambda \mathrm{Id}$;

(2)

$$
S=\left(\begin{array}{lllll}
\lambda & & & & \\
& \mu & & & \\
& & \mu & & \\
& & & \ddots & \\
& & & & \mu
\end{array}\right)
$$

with $\lambda \mu=\cos ^{2} \theta$ and if $n \geq 3$, then $T=\|T\| f_{1}$;

$$
S=\left(\begin{array}{ccccccc}
0 & & & & & & \\
& \lambda & & & & & \\
& & \ddots & & & & \\
& & & \lambda & & & \\
& & & \mu & & \\
& & & & \ddots & \\
& & & & & \mu
\end{array}\right)
$$

with $\lambda \mu=1$ and $f_{1}=T=\partial_{n+2}$.

Using Lemma 4.1, we can prove the following classification of semi-parallel hypersurfaces of $\mathbb{H}^{n} \times \mathbb{R}$.

THEOREM 4.2. Let $M^{n}$ be a semi-parallel hypersurface of $\mathbb{H}^{n} \times \mathbb{R}$. Then there are four possibilities:

(1) $n=2$ and $M^{2}$ is flat;

(2) $M^{n}$ is totally umbilical (see Theorem 3.3);

(3) $M^{n}$ is locally congruent to a rotation hypersurface for which the profile curve $\alpha$ and the axis $P^{2}$ are given by one of the following:

$$
\begin{aligned}
& \alpha(s)=\left(\cosh s, 0, \ldots, 0, \sinh s, \int \sqrt{C \cosh ^{2} s-1} d s\right), \\
& P^{2}=\operatorname{span}\left\{\partial_{1}, \partial_{n+2}\right\} ; \\
& \alpha(s)=\left(\cosh s, 0, \ldots, 0, \sinh s, \int \sqrt{C \sinh ^{2} s-1} d s\right), \\
& P^{2}=\operatorname{span}\left\{\partial_{n+1}, \partial_{n+2}\right\} ; \\
& \alpha(s)=\left(s, 0, \ldots,-\frac{1}{2 s}, \int \sqrt{C-\left(1 / 2 s^{2}\right)} d s\right), \\
&-\quad \text { with respect to }\left\{e_{1}, \ldots, e_{n+2}\right\}, P^{2}=\operatorname{span}\left\{e_{n+1}, e_{n+2}\right\} ;
\end{aligned}
$$

(4) $M^{n} \subseteq \bar{M}^{n-1} \times \mathbb{R}$ where $\bar{M}^{n-1}$ is a semi-parallel hypersurface of $\mathbb{H}^{n}$.

Conversely, all hypersurfaces given above are semi-parallel.

PROOF. Suppose that $M^{n}$ is a semi-parallel hypersurface of $\mathbb{H}^{n} \times \mathbb{R}$ with shape operator $S$. Lemma 4.1 yields that there are three possible forms of $S$ to consider. 
In the first case of Lemma $4.1, M^{n}$ is a totally umbilical hypersurface. This gives the second case of the theorem.

If we are in the second case of Lemma 4.1 and $n=2$, then $M^{2}$ is a general flat surface in $\mathbb{H}^{2} \times \mathbb{R}$. This gives the first case of the theorem.

If $n \geq 3$, then the form of $S$ is the one given in Theorem 2.2, characterizing rotation hypersurfaces. We also have that $\lambda \mu=\cos ^{2} \theta$. This is not a functional relation in the strict sense, because $\theta$ can be a nonconstant function. However, we see from (2.13) that $\theta$ does not vary in directions orthogonal to $T$. By studying [4, proof of Theorem 2], we see that this is actually enough to conclude that $M^{n}$ is a rotation hypersurface of $\mathbb{H}^{n} \times \mathbb{R}$. We now have to consider three cases, depending on whether the rotation axis $P^{2}$ is Lorentzian, Riemannian or degenerate.

Assume first that $P^{2}$ is Lorentzian and spanned by $\partial_{1}$ and $\partial_{n+2}$. From the equation $\lambda \mu=\cos ^{2} \theta$, we will be able to determine the profile curve of the rotation hypersurface $M^{n}$. From (2.17), we see that this equation is satisfied if the profile curve is a vertical line. We are then in case (4) of the theorem, where $\bar{M}^{n-1}$ is a totally umbilical hypersurface of $\mathbb{H}^{n}$. If the profile curve is not a vertical line, it can be parametrized as $\alpha(s)=(\cosh s, 0, \ldots, 0, \sinh s, a(s))$ and formulae $(2.15)$ give that

$$
\lambda \mu=\frac{a^{\prime}(s) a^{\prime \prime}(s) \operatorname{coth} s}{\left(1+a^{\prime}(s)^{2}\right)^{2}} .
$$

We also have that

$$
\cos ^{2} \theta=1-\sin ^{2} \theta=1-\left\langle\partial_{n+2}, \frac{T}{\|T\|}\right\rangle^{2}=1-\left\langle\partial_{n+2}, \frac{\alpha^{\prime}}{\left\|\alpha^{\prime}\right\|}\right\rangle^{2}=\frac{1}{1+a^{\prime}(s)^{2}} .
$$

Hence, the equation $\lambda \mu=\cos ^{2} \theta$ becomes $a^{\prime}(s) a^{\prime \prime}(s) \operatorname{coth} s=1+a^{\prime}(s)^{2}$, or, equivalently, $a^{\prime}(s)^{2}=C \cosh ^{2} s-1$, where $C$ is a real constant. This covers the first subcase of case (3) of the theorem.

Suppose now that the axis of rotation $P^{2}$ is Riemannian and spanned by $\partial_{n+1}$ and $\partial_{n+2}$. By (2.18), the equation $\cos ^{2} \theta=\lambda \mu$ is satisfied for vertical lines. We are then in case (4) of the theorem, where $\bar{M}^{n-1}$ is a totally umbilical hypersurface of $\mathbb{H}^{n}$. If the profile curve is not a vertical line, it can be parametrized as $\alpha(s)=$ $(\cosh s, 0, \ldots, 0, \sinh s, a(s))$. Using Equations (2.16), we see that $\lambda \mu=\cos ^{2} \theta$ is equivalent to $a^{\prime \prime}(s) a^{\prime}(s) \tanh s=1+a^{\prime}(s)^{2}$, or, equivalently, $a^{\prime}(s)^{2}=C \sinh (s)^{2}-1$ for some real constant $C$ and we obtain the second subcase of case (3) of the theorem.

Finally, we suppose that the rotation axis $P^{2}$ is degenerate and spanned by $e_{n+1}$ and $e_{n+2}$. By (2.20), the equation $\lambda \mu=\cos ^{2} \theta$ is satisfied if the profile curve is a vertical line. We are then in case (4) of the theorem, where $\bar{M}^{n-1}$ is a totally umbilical hypersurface of $\mathbb{H}^{n}$. If the profile curve is not a vertical line, it can be parametrized as $\alpha(s)=(s, 0, \ldots, 0,-1 / 2 s, a(s))$ with respect to the pseudo-orthonormal basis $\left\{e_{1}, \ldots, e_{n+1}, e_{n+2}\right\}$. Formulae (2.19) give that

$$
\lambda \mu=\frac{s a^{\prime}(s)\left(s a^{\prime}(s)+s^{2} a^{\prime \prime}(s)\right)}{\left(1+s^{2} a^{\prime}(s)^{2}\right)^{2}} .
$$


Combining this with

$$
\cos ^{2} \theta=\frac{1}{1+s^{2} a^{\prime}(s)^{2}}
$$

gives

$$
s^{2} a^{\prime}(s)^{2}+s^{3} a^{\prime}(s) a^{\prime \prime}(s)=1+s^{2} a^{\prime}(s)^{2},
$$

or, equivalently, $a^{\prime}(s)^{2}=C-\left(1 / 2 s^{2}\right)$ for some real constant $C$. So we obtain the third subcase of case (3) of the theorem.

In the last case of Lemma 4.1, we have that $\partial_{n+2}$ is tangent to $M^{n}$ and hence $M^{n}$ is an open part of $\bar{M}^{n-1} \times \mathbb{R}$, where $\bar{M}^{n-1}$ is a hypersurface of $\mathbb{H}^{n}$. Since $\mathbb{H}^{n}$ is a totally geodesic hypersurface of $\mathbb{H}^{n} \times \mathbb{R}$, we have that the shape operator $\bar{S}$ of $\bar{M}^{n-1}$ in $\mathbb{H}^{n}$ satisfies $\bar{S} X=S X$ for $X$ tangent to $\bar{M}^{n-1}$. It follows that $\bar{S}$ takes the form

$$
\bar{S}=\left(\begin{array}{cccccc}
\lambda & & & & & \\
& \ddots & & & & \\
& & \lambda & & & \\
& & & \mu & & \\
& & & & \ddots & \\
& & & & & \mu
\end{array}\right),
$$

with $\lambda \mu=1$. It was proven in [3] that this is the shape operator of a semi-parallel hypersurface of $\mathbb{H}^{n}$.

REMARK 4.3. We can compute the third integral appearing in case (3) of Theorem 4.2 explicitly. Since $\alpha$ cannot intersect $P^{2}$, we may assume that $s>0$. The integral is then given by

$$
\int \sqrt{C-\frac{1}{2 s^{2}}} d s=\frac{1}{\sqrt{2}}\left(\sqrt{2 C s^{2}-1}+\arctan \left(\frac{1}{\sqrt{2 C s^{2}-1}}\right)\right)+D .
$$

An explicit expression for the first two integrals involves the normal elliptic integral of the second kind.

\section{Parallel hypersurfaces in $\mathbb{H}^{n} \times \mathbb{R}$}

It is well known that a parallel hypersurface is also semi-parallel. In fact the condition of semi-parallelism is the integrability condition for the system of differential equations in the components of $h$ that expresses the condition of parallelism. However, in the case of $\mathbb{H}^{n} \times \mathbb{R}$ the classification of parallel hypersurfaces follows directly from the Codazzi equation.

THEOREM 5.1. Let $M^{n}$ be a parallel hypersurface of $\mathbb{H}^{n} \times \mathbb{R}$. Then there are two possibilities:

(1) $M^{n}$ is an open part of a totally geodesic hypersurface $\mathbb{H}^{n} \times\left\{t_{0}\right\}$; 
(2) $M^{n}$ is an open part of a Riemannian product $\bar{M}^{n-1} \times \mathbb{R}$, where $\bar{M}^{n-1}$ is a parallel hypersurface of $\mathbb{H}^{n}$.

Conversely, both types of hypersurfaces are parallel in $\mathbb{H}^{n} \times \mathbb{R}$.

PROOF. If $M^{n}$ is a parallel hypersurface of $\mathbb{H}^{n} \times \mathbb{R}$, then it follows from the Codazzi equation (2.11) that at any point of the hypersurface either $T=0$ or $\cos \theta=0$. Since $T$ and $\cos \theta$ are continuous and $\|T\|^{2}=1-\cos ^{2} \theta$, one of these must hold on the whole hypersurface. In the first case, $\partial_{n+2}$ is everywhere orthogonal to $M^{n}$ and hence $M^{n}$ is an open part of a hypersurface of type $\mathbb{H}^{n} \times\left\{t_{0}\right\}$. In the second case, we have that $\partial_{n+2}$ is everywhere tangent to $M^{n}$ and therefore $M^{n}$ is an open part of a hypersurface of type $\bar{M}^{n-1} \times \mathbb{R}$, with $\bar{M}^{n-1}$ a hypersurface of $\mathbb{H}^{n}$. It is easy to see that $\bar{M}^{n-1} \times \mathbb{R}$ is parallel in $\mathbb{H}^{n} \times \mathbb{R}$ if and only if $\bar{M}^{n-1}$ is parallel in $\mathbb{H}^{n}$.

\section{References}

[1] B. Y. Chen, Geometry of Submanifolds (Marcel Dekker, New York, 1974).

[2] B. Daniel, 'Isometric immersions into $\mathbb{S}^{n} \times \mathbb{R}$ and $\mathbb{H}^{n} \times \mathbb{R}$ and applications to minimal surfaces', Trans. Amer. Math. Soc. 361 (2009), 6255-6282.

[3] F. Dillen, 'Semi-parallel hypersurfaces of real space forms', Israel J. Math. 75 (1991), 193-202.

[4] F. Dillen, J. Fastenakels and J. Van der Veken, 'Rotation hypersurfaces in $\mathbb{S}^{n} \times \mathbb{R}$ and $\mathbb{H}^{n} \times \mathbb{R}^{\text {', }}$ Note Mat. 29 (2009), 41-54.

[5] H. B. Lawson, 'Local rigidity theorems for minimal hypersurfaces', Ann. of Math. (2) 89 (1969), 187-197.

[6] P. J. Ryan, 'A note on conformally flat spaces with constant scalar curvature', in: Proceedings of the 13th Biennial Seminar of the Canadian Mathematical Congress, Vol. 2, Canad. Math. Congr., Montreal (1972), 115-124.

[7] R. Souam and E. Toubiana, 'Totally umbilic surfaces in homogeneous 3-manifolds', Comment. Math. Helv. 84 (2009), 673-704.

[8] J. Van der Veken, 'Higher order parallel surfaces in Bianchi-Cartan-Vranceanu spaces', Results Math. 51 (2008), 339-359.

[9] J. Van der Veken and L. Vrancken, 'Parallel and semi-parallel hypersurfaces of $\mathbb{S}^{n} \times \mathbb{R}^{\prime}$, Bull. Braz. Math. Soc. 39 (2008), 355-370.

GIOVANNI CALVARUSO, Dipartimento di Matematica, Università del Salento, 'E. De Giorgi', Provinciale Lecce-Arnesano, 73100 Lecce, Italy

e-mail: giovanni.calvaruso@unile.it

DANIEL KOWALCZYK, Departement Wiskunde, Katholieke Universiteit Leuven, Celestijnenlaan, 200B, Box 2400, B-3001 Leuven, Belgium

e-mail: daniel.kowalczyk@wis.kuleuven.be

JOERI VAN DER VEKEN, Departement Wiskunde, Katholieke Universiteit Leuven, Celestijnenlaan 200B, Box 2400, B-3001 Leuven, Belgium

e-mail: joeri.vanderveken@wis.kuleuven.be 